\title{
Reflets
}

Revue ontaroise d'intervention sociale et communautaire

\section{Santé mentale et suicide des jeunes}

\section{Anselme Mvilongo et Michel-André Beauvolsk}

Volume 7, numéro 1, printemps 2001

Santé mentale et les défis de l’an 2001

URI : https://id.erudit.org/iderudit/026337ar

DOI : https://doi.org/10.7202/026337ar

Aller au sommaire du numéro

Éditeur(s)

Reflets : Revue ontaroise d'intervention sociale et communautaire

ISSN

1203-4576 (imprimé)

1712-8498 (numérique)

Découvrir la revue

Citer cet article

Mvilongo, A. \& Beauvolsk, M.-A. (2001). Santé mentale et suicide des jeunes. Reflets, 7(1), 70-89. https://doi.org/10.7202/026337ar

\section{Résumé de l'article}

Le suicide est un acte volontaire posé par un sujet individuel. Il est toutefois très souvent associé à la maladie mentale, à un déséquilibre mental, car on conçoit difficilement qu'unepersonne mentalement bien équilibrée se donne la mort.Dans cet article, les auteurs veulent attirer l'attention du lecteur et de la lectrice sur lesfacteurs intrinsèques à la personne du suicidé. Ces facteurs qui ne témoignent pas nécessairementd'un bon équilibre psychique, psychologique et d'ajustement à la réalité — ce qui pourraitdéfinir la santé mentale - naissent souvent de facteurs de vulnérabilité qui viennent del'environnement social et qui sont nourris par ces mêmes facteurs. Ces derniers portent tous eneux le germe de la maladie mentale. C'est pourquoi le suicide, bien qu'il soit un acte volontaire,est associé à la maladie mentale. On ne saurait ainsi traiter de la même manière la mort del'halluciné qui se précipite d'une fenêtre élevée parce qu'il la croit à la hauteur du sol, et cellede l'homme, sain d'esprit, qui se frappe en sachant ce qu'il fait. Les auteurs arrivent à cetteconclusion après avoir analysé deux formes de suicide pour montrer le lien entre la maladiementale et l'acte suicidaire.
Tous droits réservés (C) Reflets : Revue ontaroise d'intervention sociale et communautaire, 2001
Ce document est protégé par la loi sur le droit d'auteur. L'utilisation des services d’Érudit (y compris la reproduction) est assujettie à sa politique d'utilisation que vous pouvez consulter en ligne.

https://apropos.erudit.org/fr/usagers/politique-dutilisation/ 


\title{
Santé mentale et suicide des jeunes
}

Le suicide est un acte volontaire posé par un sujet individuel. Il est toutefois très souvent associé à la maladie mentale, à un déséquilibre mental, car on conçoit difficilement qu'une personne mentalement bien équilibrée se donne la mort.

Dans cet article, les auteurs veulent attirer l'attention du lecteur et de la lectrice sur les facteurs intrinsèques à la personne du suicidé. Ces facteurs qui ne témoignent pas nécessairement d'un bon équilibre psychique, psychologique et d'ajustement à la réalité - ce qui pourrait définir la santé mentale — naissent souvent de facteurs de vulnérabilité qui viennent de l'environnement social et qui sont nourris par ces mêmes facteurs. Ces derniers portent tous en eux le germe de la maladie mentale. C'est pourquoi le suicide, bien qu'il soit un acte volontaire, est associé à la maladie mentale. On ne saurait ainsi traiter de la même manière la mort de l'halluciné qui se précipite d'une fenêtre élevée parce qu'il la croit à la hauteur du sol, et celle de l'homme, sain d'esprit, qui se frappe en sachant ce qu'il fait. Les auteurs arrivent à cette conclusion après avoir analysé deux formes de suicide pour montrer le lien entre la maladie mentale et l'acte suicidaire.

\author{
Anselme Mvilongo et Michel-André Beauvolsk \\ École de service social, Université Laurentienne
}

\section{Introduction}

La santé mentale et le suicide sont des sujets préoccupants dans notre société depuis quelques décennies, surtout avec l'accroissement du taux alarmant de suicide chez les jeunes. Le suicide devient si commun dans les populations qu'il est immédiatement associé à la maladie mentale, tout comme l'on fait de la plupart des meurtriers à qui on fait subir d'emblée un examen psychiatrique. Mais, puisque le suicidé n'est plus, l'on se contente 
"..." elle ne devait pas être bien mentalement...».» de dire de la personne : «elle ne devait pas être bien mentalement...». Dans un premier temps, nous voulons tout d'abord clarifier les notions de santé (maladie) mentale et de suicide. Dans un deuxième temps, en clarifiant ces concepts, nous découvrirons les liens entre les différents types de suicide et la maladie mentale, ainsi que ce qui les distingue. En troisième lieu, nous décrirons un cas pratique pour montrer que les facteurs externes de vulnérabilité du sujet réputé malade mental sont parfois les mêmes qui conduisent au suicide. Nous conclurons par quelques réflexions susceptibles de guider une intervention pratique en travail social.

Puisqu'un des buts de ce travail est de clarifier les concepts de santé (ou de maladie) mentale, nous abordons ces concepts en les définissant de façon descriptive. Nous analysons ensuite les formes de suicide pour montrer la présence de facteurs intra-psychiques et extra-psychiques ou sociaux dans l'acte de suicide, et pour voir que les facteurs externes de vulnérabilité sont de même nature pour le suicide que pour la maladie mentale. Nous illustrerons ceci par l'analyse d'un cas concret pour montrer le lien entre la maladie mentale et le suicide.

\section{Concepts de santé mentale et de suicide : une définition}

« «Ma vie m'appartient; j'ai le droit d'en disposer comme je veux. " "
«Ma vie m'appartient;j'ai le droit d'en disposer comme je veux.» Nous entendons souvent cette phrase, même de la part d'enfants. Par conséquent, s'enlever la vie est un acte qui relève de la seule volonté, de la seule décision du sujet. Un tel acte, quand il se réalise, s'appelle le suicide; il pourrait se définir comme tout comportement qui cherche et trouve une solution pour un problème existentiel, dans le fait d'attenter à sa vie. Cet acte est donc supposé être celui d'un individu jouissant d'un parfait équilibre de toutes ses facultés de cœur, d'esprit, bref, d'une santé mentale; cette dernière est définie comme un équilibre psychique et psychologique chez une personne à un moment donné. Cet 
équilibre s'apprécie, entre autres, à l'aide des éléments suivants :le niveau de bien-être subjectif, l'exercice des capacités mentales et la qualité des relations avec le milieu (Corin et al. 1985).

\section{Clarification des liens}

"Il importe de clarifier les liens dynamiques entre santé mentale et suicide, ce qu'ils ont en commun et ce qui les distingue. »
Cette définition dynamique de la santé mentale est le résultat d'interactions, entre autres, des facteurs contextuels, qui ont trait aux relations entre la personne et son environnement. Mais quand un individu en vient à s'enlever le plus précieux bien qu'il possède, la vie, est-il toujours vrai que son interaction avec son environnement social est en parfait équilibre et n'est pas troublée de quelque façon? Il faut noter, selon Bibeau et al. (1992), que toute condition qui nuit à l'adaptation réciproque de la personne et de son milieu constitue un obstacle à la santé mentale. Or, les obstacles à la santé mentale peuvent être constitués de facteurs intrinsèques ou internes à l'individu, tout comme ils peuvent venir de l'extérieur

Il importe de clarifier les liens dynamiques entre santé mentale et suicide, ce qu'ils ont en commun et ce qui les distingue. Tout d'abord, le suicide n'est pas une maladie à proprement parler (même si l'on voulait suggérer une pathologie sociale), dans une société, comme le serait une épidémie que l'on pourrait enrayer ou endiguer par la vaccination préventive pour préserver la santé publique dans la société.

Alors que la santé mentale est le résultat d'interactions positives de facteurs contextuels ayant trait aux relations entre la personne et son environnement, l'acte de suicide est le résultat d'interactions négatives, ou même du manque d'interactions, entre le suicidé et son environnement social. La santé mentale réside avant tout dans un individu, mais c'est un bien qui se répand et dont bénéficie tout son entourage; la mort par suicide ne se répand pas nécessairement, elle reste un fait individuel, même si l'entourage en souffre les conséquences. Par ailleurs, la société voudrait savoir que tous ses membres jouissent d'une parfaite santé mentale, et 
"Si le fait de s'enlever la vie est un comportement qui cherche et trouve une solution pour un problème existentiel, le suicide n'est donc pas un acte fortuit, un accident. Il est le résultat d'un acte choisi, planifié dans le temps...» qu'aucun ne perde sa vie par suicide. Le suicide à l'adolescence est un phénomène dont la croissance devient de plus en plus dramatique dans la société. Au Canada, il constitue présentement la deuxième cause de mortalité chez les jeunes.

Parmi les formes de suicide, nous distinguerons celles qui sont le plus susceptibles d'être pratiquées par les jeunes. Là encore, le lien entre la maladie ou le manque de santé mentale et le suicide se clarifiera davantage. Si le fait de s'enlever la vie est un comportement qui cherche et trouve une solution pour un problème existentiel, le suicide n'est donc pas un acte fortuit, un accident. Il est le résultat d'un acte choisi, planifié dans le temps et dans l'espace ou dans l'environnement social qui en est, si non la cause immédiate, du moins la cause éloignée. Ceci soulève certaines questions. Le suicide est-il seulement la mort d'un individu qui a décidé de s'enlever la vie? Si non, quels sont les facteurs qui mènent au suicide? Examinons d'abord certains types de suicide. À travers eux, nous verrons peut-être le niveau de comportement ou de trouble mental de l'individu qui se suicide.

\section{Formes de suicide}

Témoins de plusieurs morts par suicide dans notre vie professionnelle, nous nous sommes toujours demandés pourquoi une personne qui paraissait lucide et sereine le matin se tuait le soir en se jetant du haut du pont Jacques-Cartier à Montréal, en se précipitant devant une voiture en vitesse ou en se tirant une balle à la tête, etc. Si tous ces décès sont le fait d'une personne qui préfere la mort à la vie, les causes qui les déterminent ne sont pas de même nature dans tous les cas : elles sont même, parfois, opposées entre elles. Or, il est impossible que la différence des causes ne se retrouve pas dans les effets. On peut donc être certain qu'il $\mathrm{y}$ a plusieurs sortes de suicides qualitativement distinctes les unes des autres. Mais les causes profondes du suicide restent connues des seuls suicidés et nous ne pouvons appréhender que les effets externes qui en dérivent. C'est donc dire que les mobiles 
"Le modèle épidémiologique le plus souvent utilisé essaie d'identifier les facteurs de risque qui appartiennent à la biologie des victimes."

\section{"La recherche} contemporaine s'intéresse plus à l'interaction entre des facteurs individuels et des facteurs sociaux, mais si l'on maintient que le suicide est un acte volontaire, il devient important de regarder la dynamique des facteurs internes ou intra-psychiques de l'individu pour qualifier le modèle de suicide qu'il accomplit. " intrinsèques au sujet qui se tue lui-même demeurent insaisissables. C'est pourquoi beaucoup de chercheurs contemporains et d'associations dans la communauté s'intéressent davantage à l'interaction entre les facteurs individuels et les facteurs sociaux (Tousignant 1995; Bouchard s.d.; Dossier Hommes et Suicide 1999; Dulac 1999; Chagnon 1999; Chabot 1999; Association québécoise de suicidologie 1999; Dyck et al. 1999).

Le modèle épidémiologique le plus souvent utilisé essaie d'identifier les facteurs de risque qui appartiennent à la biologie des victimes. Parmi ces facteurs, on peut encore distinguer les agents précipitants et les traits de vulnérabilité. Ces facteurs de vulnérabilité ne sont pas des causes directes; ils augmentent la probabilité du suicide lorsque des circonstances adverses, comme une mauvaise estime de soi ou le manque de soutien social adéquat, se présentent. D'autres facteurs de vulnérabilité peuvent remonter à l'enfance et sont les conséquences de mauvais traitements et de négligence de la part des parents. Mais un agent précipitant est souvent à la base et il est presque toujours accompagné d'un ou de plusieurs facteurs de vulnérabilité. Si les peines d'amour conduisent parfois à des tentatives de suicide chez les jeunes, c'est souvent à cause d'un manque de vécu dans la relation avec les parents qui les rend plus fragiles lors des pertes subies plus tard. Le modèle épidémiologique est populaire auprès des milieux scientifiques parce qu'il se prête bien à une validation empirique, mais il parle peu de la dynamique macro-sociale qui entoure les événements individuels. Il s'avère bien impuissant encore à expliquer les mobiles internes de l'individu qui se donne la mort.

La recherche contemporaine s'intéresse plus à l'interaction entre des facteurs individuels et des facteurs sociaux, mais si l'on maintient que le suicide est un acte volontaire, il devient important de regarder la dynamique des facteurs internes ou intra-psychiques de l'individu pour qualifier le modèle de suicide qu'il accomplit. Durkheim (1969) distinguait déjà trois formes principales de suicide : égoïste, anomique et altruiste. Selon lui, le suicide égoïste provient du fait que les institutions comme la religion, la famille, l'État peuvent exercer un attrait variable sur leurs membres et que la trop grande liberté individuelle fait en sorte que la personne 
ne se considère plus liée par ses responsabilités à l'égard de la société et qu'elle peut s'enlever la vie. Ce qui distingue l'égoïste qui se tue, c'est une dépression générale qui se manifeste soit par une langueur mélancolique, soit par l'indifférence épicurienne.

Par ailleurs, l'affaiblissement des normes et du contrôle des institutions conduit au suicide anomique.Toujours selon Durkheim (1969), il est une troisième sorte de suicidés qui s'opposent aux premiers en ce que leur acte est essentiellement passionnel :la passion qui les inspire et qui domine la scène finale est d'une toute autre nature. Il ne s'agit pas d'enthousiasme, de foi religieuse, morale ou politique, ni d'aucune des vertus militaires, mais de colère et de tout ce qui, d'ordinaire, accompagne la déception.

Le suicide altruiste, parce qu'il a pour origine un sentiment violent, ne va pas sans un certain déploiement d'énergie. Dans le cas du suicide obligatoire, cette énergie est mise au service de la raison et de la volonté. Le sujet se tue parce que sa conscience le lui ordonne; il se soumet à un impératif.Aussi son acte a-t-il pour note dominante cette fermeté sereine que donne le sentiment du devoir accompli; la mort de Caton en est le type historique.

Même teintés d'une certaine connotation morale, les concepts de Durkheim demeurent source d'inspiration pour essayer de comprendre certains suicides dont on aurait été témoins. Les comportements observés, particulièrement chez les jeunes, émanent de certains états intra-psychiques :l'isolement, le désintéressement de toute activité, par exemple. Une telle analyse pourrait être une autre avenue de réflexion sur le drame du suicide des jeunes. Ce comportement n'est pas nouveau, même s'il apparait plus frappant chez beaucoup de personnes aujourd'hui et s'il choque davantage quand il se produit chez les jeunes. En effet, il est une première forme de suicide que l'Antiquité a certainement connue, mais qui s'est surtout développée de nos jours :le Raphaël de Lamartine en offre le type idéal.

Cette forme de suicide se caractérise par un état de langueur mélancolique chez la personne, langueur qui détend les ressorts de l'action. Les affaires, les fonctions publiques, le travail utile, même les devoirs domestiques n'inspirent au sujet qu'indifférence et éloignement. Le jeune répugne à sortir de lui-même. En 
revanche, la pensée et la vie intérieure gagnent tout ce que perd l'activité. En se détournant de ce qui l'entoure, la conscience se replie sur elle-même, se prend elle-même comme son propre et unique objet et se donne pour principale tâche de s'observer et de s'analyser. Mais par cette extrême concentration, elle ne fait que rendre plus profond le fossé qui la sépare du reste de l'univers.

Du moment où l'individu s'éprend à ce point de soi-même, il ne peut que se détacher davantage de tout ce qui n'est pas lui et consacrer, en le renforçant, l'isolement dans lequel il vit. Ce n'est pas en ne regardant que soi qu'on peut trouver des raisons de s'attacher à autre chose que soi. Tout mouvement, en un sens, est altruiste, car il est centrifuge et répand l'être hors de lui-même. La réflexion, au contraire, a quelque chose de personnel et d'égoïste; car elle n'est possible que dans la mesure où le sujet se dégage de l'objet et s'en éloigne pour revenir sur soi-même, et elle est d'autant plus intense que ce retour sur soi est plus complet. On ne peut agir qu'en se mêlant au monde; pour le penser, au contraire, il faut cesser d'être confondu avec lui, de manière à pouvoir le contempler $\mathrm{du}$ dehors et, à plus forte raison, pour se penser soi-même. Celui dont toute l'activité se tourne en pensée intérieure devient insensible à tout ce qui l'entoure. S'il aime, ce n'est pas pour se donner, pour s'unir, dans une union féconde, à un autre être que lui; c'est pour méditer sur son amour. Ses passions ne sont qu'apparentes; car elles sont stériles. Elles se dissipent en vaines combinaisons d'images, sans rien produire qui leur soit extérieur.

En revanche, toute vie intérieure tire du dehors sa matière première. Nous ne pouvons penser que des objets ou la manière dont nous les pensons. Nous ne pouvons pas réfléchir à notre conscience dans un état d'indétermination pure; sous cette forme, elle est impensable. Or, elle ne se détermine qu'affectée par autre chose qu'elle-même. Si donc elle s'individualise au-delà d'un certain point, si elle se sépare trop radicalement des autres êtres, hommes ou choses, elle se trouve ne plus communiquer avec les sources mêmes auxquelles elle devrait normalement s'alimenter et n'a plus rien à quoi elle puisse s'appliquer. En faisant le vide autour d'elle, elle a fait le vide en elle et il ne lui reste plus rien auquel réfléchir que sa propre misère. Elle n'a plus pour objet de 
méditation que le néant qui est en elle et la tristesse qui en est la conséquence. Elle s'y complait, s'y abandonne avec une sorte de joie maladive que Lamartine (1960) a merveilleusement décrite par la bouche de son héros : Raphäl.

La langueur de toutes choses autour de moi était,dit$i l$, une merveilleuse consonance avec ma propre langueur. Elle l'accroissait en la charmant. Je me plongeais dans des abîmes de tristesse. Mais cette tristesse était vivante, assez pleine de pensées, d'impressions, de communications avec l'infini, de clairobscur dans mon âme pour que je ne désirasse pas m'y soustraire. Maladie de l'homme, mais maladie dont le sentiment même est un attrait au lieu d'être une douleur, et où la mort ressemble à un voluptueux évanouissement dans l'infini.J'étais résolu à m'y livrer désormais tout entier, à me séquestrer de toute société qui pouvait m'en distraire, et à m'envelopper de silence, de solitude et de froideur, au milieu du monde que je rencontrerais là; mon isolement d'esprit était un linceul à travers lequel je ne voulais plus voir les hommes, mais seulement la nature et Dieu.

"Quand une personne éprouve tant de plaisir à n'être pas, elle ne peut satisfaire complètement son penchant qu'en renonçant complètement à être.»
Mais on ne saurait rester ainsi en contemplation devant le vide, sans y être progressivement attiré. On aura beau le décorer du nom d'infini, il ne change pas pour cela de nature. Quand une personne éprouve tant de plaisir à n'être pas, elle ne peut satisfaire complètement son penchant qu'en renonçant complètement à être. Le parallélisme souvent observé entre le développement de la conscience et l'affaiblissement de vouloir vivre se trouve ainsi être exact

C'est que l'idée et le mouvement sont, en effet, deux forces antagonistes qui progressent en sens inverse l'une de l'autre, et que le mouvement, c'est la vie. «Penser, a-t-on dit, c'est se retenir d'agir»; c'est donc, dans la même mesure, se retenir de vivre. Aussi le règne absolu de l'idée ne peut-il s'établir ni surtout se maintenir, autrement c'est la mort. Il ne faudrait pas entendre par là que la réalité soit, par elle-même, intolérable, à moins d'être voilée par 
«La tristesse... Elle est un produit de notre propre pensée. "

" "Je ne veux pas montrer que je suis plus courageux ou plus lâche; je veux tout simplement utiliser le peu d'instants qui me restent à décrire les sensations que j'éprouve en m'asphyxiant et la durée de mes souffrances." " l'illusion. La tristesse n'est pas inhérente aux choses; elle ne nous vient pas du monde et par cela seul que nous le pensons. Elle est un produit de notre propre pensée. C'est le sujet qui la crée, mais il faut pour cela que la pensée du sujet soit anormale. Si parfois la conscience fait le malheur de l'être humain, c'est seulement quand elle atteint un développement maladif, quand, s'insurgeant contre sa propre nature, elle se pose comme un absolu et cherche en elle-même sa propre fin.

Le stoïcisme lui aussi enseigne que l'homme doit se détacher de tout ce qui est extérieur pour vivre de lui-même et par luimême. Seulement, comme la vie se trouve alors sans raison, la doctrine conclut au suicide.

Le jeune garçon (18 ans) de mes amis s'était asphyxié dans sa petite cabane au fond de leur cour en soufflant avec la bouche le feu de bois dont la fumée devait le tuer; il écrivait en même temps ses observations : «Je ne veux pas montrer que je suis plus courageux ou plus lâche; je veux tout simplement utiliser le peu d'instants qui me restent à décrire les sensations que j'éprouve en m'asphyxiant et la durée de mes souffrances.» Cette particularité et d'autres semblables se rattachent toutes, dans leur diversité, au suicide égoïste. On ne saurait douter qu'elles n'en soient la conséquence et l'expression individuelle. La paresse à l'action dont il a été fait précédemment mention et le détachement mélancolique résultent de cet état d'individuation exagéré par lequel se définit ce type de suicide.

Si l'individu s'isole, c'est parce que les liens qui l'unissent aux autres êtres autour de lui sont détendus ou brisés, c'est parce que la société, sur les points où il est en contact avec elle, n'est pas assez fortement intégrée. Les vides qui séparent les consciences et les rendent étrangères les unes aux autres viennent précisément du relâchement du tissu social. Enfin, le caractère intellectuel et méditatif de ces sortes de suicides pourrait facilement s'expliquer, si l'on se rappelle que le suicide égoïste a pour accompagnement nécessaire un grand développement de la science et de l'intelligence réfléchie. Il apparait évident, en effet, que dans une société où la conscience est normalement poussée à étendre son champ d'action, elle soit aussi beaucoup plus exposée à excéder ces limites normales qu'elle ne peut dépasser sans se détruire elle-même. 
Une pensée qui met tout en question, si elle n'est pas assez ferme pour porter le poids de son ignorance, risque de se mettre elle-même en question et de s'abîmer dans le doute. Car, si elle ne parvient pas à découvrir les titres que peuvent avoir à l'existence les choses sur lesquelles elle s'interroge, elle leur déniera toute réalité. Le seul fait qu'elle pose le problème implique déjà qu'elle penche vers les solutions négatives. Mais, du même coup, elle se videra de tout contenu positif et, ne trouvant plus rien devant elle qui lui résiste, elle ne pourra plus que se perdre dans le vide des rêveries intérieures.

Cette forme élevée du suicide égoïste n'est pas la seule; il en est une autre, plus vulgaire. Au lieu de méditer tristement sur son état, le sujet en prend allégrement son parti. Il est conscient de son égoïsme et des conséquences qui en découlent logiquement, mais il les accepte à l'avance et entreprend de vivre comme un enfant, avec cette seule différence qu'il se rend compte de ce qu'il fait. Il se donne donc comme tâche unique de satisfaire ses besoins personnels, les simplifiant même pour en rendre la satisfaction plus assurée. Sachant qu'il ne peut rien espérer d'autre, il est tout disposé, s'il est empêché d'atteindre cette unique fin, à se défaire d'une existence désormais sans raison. C'est le suicide épicurien. Épicure n'ordonnait pas à ses disciples de hâter la mort, il leur conseillait, au contraire, de vivre tant qu'ils y trouvaient quelque intérêt. Seulement sentant bien que si l'on n'a pas d'autre but, qu'on est à chaque instant exposé à ne plus en avoir aucun, et que le plaisir sensible est un lien bien fragile pour rattacher l'homme à la vie, il les exhortait à se tenir toujours prêts à en sortir, au moindre appel des circonstances. Ici la mélancolie

"...une personne jouissant d'un parfait équilibre physique, psychique, psychologique et mental peut-elle s'enlever la vie? La réponse normalement serait non!» philosophique et rêveuse est remplacée par un sang-froid sceptique et désabusé qui est particulièrement sensible à l'heure du dénouement. Le patient se frappe sans haine, sans colère, mais aussi sans cette satisfaction morbide avec laquelle l'intellectuel savoure son suicide.

Une question qui vient spontanément à l'esprit est la suivante : une personne jouissant d'un parfait équilibre physique, psychique, psychologique et mental peut-elle s'enlever la vie? La réponse normalement serait non! C'est pourquoi devant un suicide, la 
réaction première est de penser que la personne n'était pas normale; nous pensons même qu'elle était malade ou du moins qu'elle souffrait d'un déséquilibre psychique, psychologique, affectif ou d'ajustement social. Le suicide est ainsi associé à la maladie mentale.

\section{Causes indirectes du suicide}

La mélancolie philosophique ou le repli sur soi dont nous avons parlé précédemment dans le suicide égoïste tire son origine non seulement des facteurs intra-psychiques, mais aussi de plusieurs facteurs sociaux; mieux encore, ces derniers sont réalisateurs tout comme ils sont générateurs des premiers. L'acte final de suicide est la synthèse de multiples influences de ces facteurs les uns sur les autres. Parmi les causes indirectes, soulignons la solitude et l'impuissance des jeunes.

Les jeunes sont seuls! Pourtant la jeunesse est si magnifiée dans la société contemporaine : la jeunesse est belle, elle est forte, elle est pleine d'idées, elle est productive. Pourtant, dans un livre dressant le portrait de collégiens américains, When Dreams and Heroes Die, Arthur Levine (1980) les décrivait comme des voyageurs de première classe sur le Titanic. Qu'est-ce à dire? Les jeunes travaillent dur pour se bâtir un îlot de sécurité dans un monde où ils anticipent l'aggravation des problèmes et où ils se sentent impuissants.

Voilà, assez bien résumé, l'un des drames à la fois profond et complexe de la jeunesse actuelle : plusieurs ressentent une défiance, avouée ou non, à l'égard des adultes et de ses institutions, une grande solitude et une impuissance. Et, selon Lefebvre (1998), nous touchons ici une dimension proprement existentielle et spirituelle de l'expérience des jeunes. Bien sûr, ils peuvent dire croire en une foule de choses, mais c'est d'abord sur le plan de l'expérience humaine la plus vitale que leur «incroyance» se révèle étouffée sous les expressions : «Je vais me débrouiller», «Je ne sais 
« «Cet éloge funèbre révèle l'indigence d'une civilisation qui n'a plus d'autres modèles à offrir à sa jeunesse que ses idoles, morts [sic] de préférence... Ainsi, on entretient une esthétique de désespoir dont le cri de ralliement - No Future - permet de démasquer l'indifférence et l'enrichissement de quelques uns..." pas»... Il est difficile aujourd'hui de croire aux autres et à travers ceux-ci au monde, à la société, à l'amour, à la justice, à l'avenir.

Notre société est assez bien équipée pour comprendre et accompagner les jeunes, surtout la phase adolescente, mais la vingtaine, qui se situe dans le prolongement de cette dernière, demeure une étape psychique peu explorée et peu comprise. Les adolescents vivent de fortes sensations de stress, de confusion, de doute de soi, une pression pour être meilleur, des incertitudes financières et beaucoup d'autres peurs encore. Le sentiment ou la peur d'exclusion, renforcé par une dépendance de plus en plus longue, l'absence de responsabilités sociales et économiques réelles et valorisantes, peuvent être la source de pensées ou d'actes suicidaires. "On n'a pas de lieu pour s'exprimer, pour agir réellement, alors on tourne notre agressivité contre nous-mêmes», avons-nous souvent entendu de la part des jeunes.

Une crise des modèles significatifs n'est pas étrangère à ce repli sur soi. Commentant le suicide de Kurt Cobain, Caccia écrivait dans Le Devoir (5 mai 1994) : "Cet éloge funèbre révèle l'indigence d'une civilisation qui n'a plus d'autres modèles à offrir à sa jeunesse que ses idoles, morts [sic] de préférence... Ainsi, on entretient une esthétique de désespoir dont le cri de ralliement - No Future - permet de démasquer l'indifférence et l'enrichissement de quelques uns... Comment? En faisant équivaloir la révolte existentielle à la mort. Exit la manifestation. Haro sur les contestations qui ne servent à rien.» La contestation demeure pourtant l'un des ressorts de l'appartenance et de la découverte de son rôle dans la cité.

Une enquête américaine, dans les années 1980, a montré que le plus haut taux de suicide était détenu par la région du sudouest incarnant «le rêve américain». On a donc eu raison de parler du «mythe californien» qui fait ses ravages : jeunesse, beauté, santé parfaite, richesse, liberté sans limite. La majorité de la population qui ne correspond pas à ces critères olympiens a peine à assumer ses frustrations. Il n'est pas exagéré de faire un lien entre ces aspirations frustrées et un certain nombre d'attitudes répandues à plus ou moins large échelle : la violence contre les autres (délinquance) ou contre soi-même (suicide), l'intolérance face à sa 
"...Durkheim avait observé un accroissement $d u$ suicide lorsque survenaient des changements brusques de condition économique, soit une flambée de prospérité ou une vague de précarité soudaine." propre souffrance ou celle des autres (maladie, handicap), l'usage de drogues qui donne l'illusion de toute-puissance et de supertrip permanent (nirvana), les croyances magiques du genre «tout est possible», «le mal ne peut pas m'atteindre». Le décrochage scolaire et le culte de la performance s'y rattachent aussi. L'école, en effet, pose des limites à la liberté, à l'acquisition des biens matériels «tout de suite», et au plaisir. En n'exaltant, par ailleurs, que les médaillés d'or, on crée chez les autres un douloureux sentiment d'être "perdants» à tout coup.

Qu'est-ce que ces attitudes ont en commun? La négation de la limite. Claude Lévi-Strauss, dans ses écrits anthropologiques, avait identifié trois piliers de la culture : la règle ou la loi qui régit les rapports et établit leurs limites, la réciprocité ou l'échange social, et le don par lequel les individus deviennent interdépendants et collaborateurs. Ces trois piliers composent une culture au sens profond, c'est-à-dire un milieu qui permet de nourrir une saine et signifiante relation aux autres. Or, qu'est-ce qui initie un jeune à la culture? C'est notamment la fonction symbolique.

Pour arriver à s'inscrire dans l'échange social, l'être humain doit passer par l'imaginaire où il se trouve enfermé dans son image et ses rêves inaccessibles à l'ordre symbolique (règle, échange, don) par la médiation des symboles. Par le langage et les images qui composent cet ordre, et qui sont reliés à la culture qui le porte, l'individu "entre dans l'ordre symbolique», est initié aux règles du jeu de la lutte et de la satisfaction, de la loi et du bonheur, de la mort et de la vie, de la réciprocité et du don.

Or, le jeune a pratiquement de la misère à entrer dans cet ordre symbolique de la société et pour cause. Non pas seulement à cause de son égoïsme, mais parce que la société contemporaine si fragmentée est fermée à ses aspirations. Le problème des aspirations non satisfaites joue sans doute un rôle très grand dans le «mal-être» de plusieurs individus. Se penchant sur les statistiques de l'époque, Durkheim avait observé un accroissement du suicide lorsque survenaient des changements brusques de condition économique, soit une flambée de prospérité ou une vague de précarité soudaine. C'est par exemple en ce sens que l'on peut faire une lecture sociale de l'augmentation du suicide. L'histoire 
"Depuis les années 1980 en particulier, nombre de jeunes doivent affronter la précarité alors qu'ils ont intégré des aspirations très fortes. " canadienne est, pour le moins, houleuse : se succèdent, depuis une quarantaine d'années, et avec rapidité, une prospérité d'aprèsguerre et une enfilade de crises économiques, et ce, sur le fond du passage très brutal d'une société au mode de vie traditionnel, encadrée par le catholicisme, à une société sécularisée, émancipée de la tutelle religieuse.

Depuis les années 1980 en particulier, nombre de jeunes doivent affronter la précarité alors qu'ils ont intégré des aspirations très fortes. Ces situations sont de nature à faire chavirer même les têtes les mieux charpentées sur les épaules. L'équilibre de vie est brisé; la santé mentale de la population en prend un coup. Dans ces conditions, on reconnait que le suicide ne relève pas que de la décision et du malheur individuels, mais de causes communes à retracer dans les domaines social, culturel et religieux. Il résulte d'un bris d'équilibre mental. Le suicide est à considérer comme un fait social. Pour illustrer cela, il suffit de rappeler une discussion lors d'un séminaire sur le sujet, tenu à l'Université du Ghana, à Accra/Legon, il y a quelques années. Le séminaire avait été suscité par un événement qui avait bouleversé la population de la ville : un jeune homme s'était suicidé. Pour beaucoup de gens, cela semblait impensable, malgré la misère, la misère très grande. Durkheim avait déjà observé, de fait, que la misère (qui engendre la maladie mentale) protégeait du suicide.

Cette conclusion a été raffinée et révisée depuis. Dans les pays occidentaux et nord-américains, les couches de la population plus démunies sont parfois touchées davantage par le suicide. Il est probable que la misère protège, dans la mesure où les gens ne souffrent pas d'un écart trop grand entre leurs aspirations et les moyens de les satisfaire. Elle protège là aussi où la religion demeure une référence collective forte et donne de l'espoir aux pauvres de quelque façon. Nous avons déjà signalé que la question des aspirations non satisfaites joue un grand rôle dans le «mal-être» de plusieurs individus. Or, ne pas se sentir dans sa peau, c'est ne pas jouir de son parfait équilibre mental. Si l'on maintient que le suicide est un acte volontaire de l'individu, il reste que cet acte peut être commandé par des facteurs sociaux de vulnérabilité. 
Or, ces causes, nous les connaissons. Nous les avons déterminées quand nous avons fait voir de quelles sources découlent les principaux courants suicidogènes. Cependant, il en est un qui n'est certainement pour rien dans le progrès actuel du suicide; c'est le courant altruiste. Aujourd'hui, en effet, il perd du terrain beaucoup plus qu'il n'en gagne. S'il se maintient dans l'armée, il ne semble pas qu'il y ait une intensité anormale, car il est nécessaire, dans une certaine mesure, à l'entretien de l'esprit militaire. Le suicide égoïste et le suicide anomique sont donc les seuls dont le développement puisse être envisagé comme morbide; eux seuls peuvent être associés à la maladie mentale. Le suicide égoïste vient de ce que la société n'a pas sur tous les points une intégration suffisante pour maintenir tous ses membres sous sa dépendance. Si donc il se multiplie de façon dramatique, comme nous le déplorons chez les jeunes, c'est que cet état dont il dépend s'est lui-même répandu à l'excès; c'est que la société, troublée et affaiblie, laisse échapper trop complètement à son action un trop grand nombre de sujets. Mais comment remédier au mal? Le cas suivant illustre très brièvement la complexité du suicide.

\section{Le suicide de Suzanne}

Suzanne, âgée de 21 ans, avait toujours rêvé devenir soldat dans les Forces Armées. Elle s'était enrôlée et se trouvait à parfaire son entraînement dans une des bases canadiennes. Elle était venue me voir parce que sa relation amoureuse avec son ami, Sylvain (22 ans), n'allait pas très bien. Ce qui l'ennuyait le plus, c'étaient les rituels de fin de semaine. Lorsque le vendredi arrivait, les hommes sur la base se rencontraient au bar pour une bonne bière; ces réjouissances étaient réservées aux hommes et ne se vivaient qu'entre eux. À cause de cela, elle se sentait non respectée et même rejetée par son ami puisqu'il ne répondait pas à ses besoins de présence et de découverte. Je lui ai donc demandé s'il était possible d'inviter son ami à la prochaine session. Il refusa, prétendant qu'il ne voyait pas l'utilité de l'accompagner à sa 
"Dans sa famille, la qualité de présence à l'autre était devenue une qualité essentielle dans toutes leurs relations humaines."

"De nombreux facteurs extrapsychiques ont joué ici un rôle à la fois d'enrichissement et d'appauvrissement. " prochaine session de counseling. C'est la fin de semaine suivante que Suzanne prit une surdose de médicaments et elle est morte.

Nul n'est jamais prêt à recevoir ce genre de coup dur de la vie. Suzanne, dans un instant de dépression, de solitude, avait posé un geste qui, en soi, ne pouvait changer le comportement de son ami. Le geste soudain et sans avertissement avait transformé la vie de Suzanne, de son ami et de son conseiller. La lettre qu'elle a laissée sur son bureau mentionnait qu'elle avait un très grand amour pour Sylvain mais qu'elle n'avait pu accepter qu'il aille passer une fin de semaine à Toronto avec ses amis sans même penser à l'amener elle aussi.

Suzanne venait d'une famille de quatre enfants. Elle avait une soeur plus âgée, Julie (23 ans), et deux frères, Jacques (25 ans) et Armand (27 ans). Suzanne était donc la plus jeune de sa famille. Elle se sentait très proche de son père qui l'amenait aussi souvent qu'il le pouvait, soit visiter ses tantes et oncles, soit magasiner. Dans sa famille, la qualité de présence à l'autre était devenue une qualité essentielle dans toutes leurs relations humaines.

Suzanne nous présente donc une situation de suicide pour une valeur fondamentale dans la vie de cette personne. Elle n'explique pas tout le contexte familial dans ses valeurs et rituels fondamentaux. Il existera toujours un élément de mystère dans un tel geste. Pourquoi cela est il arrivé à ce moment là? Nul ne le saura jamais.

De nombreux facteurs extra-psychiques ont joué ici un rôle à la fois d'enrichissement et d'appauvrissement. Suzanne s'attendait retrouver chez son ami Sylvain le même support et la même chaleur qu'elle avait reçus de son père. Au plan des facteurs intrapsychiques, Suzanne croyait fortement à la valeur fondamentale de l'amour et de la qualité de présence à l'autre. Mais dans son être physique et émotionnel, Suzanne voyait la réalité comme froide et aliénante. Sa dépressivité redéfinissait négativement plusieurs événements de sa vie quotidienne. Mourir pour être aimée semblait définir sa réponse, même si sa famille l'aimait toujours. Le contexte social n'a pu l'aider à faire face à sa peine et à trouver une réponse à ses besoins intimes d'appartenance à quelqu'un de particulier. Ce qui s'est passé en elle, Sylvie est la 
seule à le savoir vraiment. Notre réflexion se base sur l'observation et l'analyse d'une courte relation thérapeutique. La santé mentale de Suzanne a été affaiblie par certains éléments de sa vie et son cri à l'aide n'a pas été vraiment entendu. Et son choix a été de terminer sa vie. Cet événement n'a pas encore affecté, semble-til, notre tabou face à la santé mentale et au suicide.

\section{Quelques réflexions}

"Il faut que

l'individu se sente davantage solidaire d'un être collectif qui l'ait précédé dans le temps, qui lui survive et qui le déborde de tous les côtés. »
Nous suggérons que la seule façon de remédier au mal est de rendre aux groupes sociaux assez de consistance pour qu'ils tiennent plus fermement l'individu et que lui-même tienne à eux. Il faut que l'individu se sente davantage solidaire d'un être collectif qui l'ait précédé dans le temps, qui lui survive et qui le déborde de tous les côtés.

À cette condition, il cessera de chercher en soi-même l'unique objet de sa conduite et, comprenant qu'il est l'instrument d'une fin qui le dépasse, il s'apercevra qu'il sert à quelque chose. La vie reprendra un sens à ses yeux parce qu'elle retrouvera son orientation et son but naturels. Mais quels sont les groupes les plus aptes à rappeler perpétuellement l'homme à ce salutaire sentiment de solidarité? Nous ne pensons pas que ce soit nécessairement la société politique. Aujourd'hui surtout, dans nos grands États modernes, elle est trop loin de l'individu pour agir efficacement sur lui avec assez de continuité. Il faut des circonstances exceptionnelles, comme une grande crise nationale ou politique pour que l'idée de la patrie passe au premier plan, envahisse les consciences et devienne le mobile directeur de la conduite. Or, ce n'est pas une action aussi intermittente qui peut refréner d'une manière régulière le penchant au suicide. Il est nécessaire que, non seulement de loin en loin, mais à chaque instant de sa vie, l'individu puisse se rendre compte que ce qu'il fait va vers un but. Pour que son existence ne lui paraisse pas vaine, il faut qu'il la voie, d'une façon constante, comme servant à une fin qui le touche immédiatement. 
Mais cela n'est possible que si un milieu social, plus simple et moins étendu, l'enveloppe de plus près et lui offre un terme plus prochain à son activité : soit la famille dont la vertu prophylactique n'est pas douteuse. Mais ce serait une illusion de croire qu'il suffit de multiplier les mariages pour arrêter le développement du suicide. C'est que, en effet, il s'est produit dans la constitution de la famille des changements qui ne lui permettent plus d'avoir la même influence préservatrice qu'autrefois. Elle n'a plus aujourd'hui qu'une durée éphémère, tandis que jadis elle maintenait la plupart de ses membres dans son orbite depuis leur naissance jusqu'à leur mort et formait une masse compacte, indivisible, douée d'une sorte de pérennité. Maintenant, à peine est-elle constituée qu'elle se disperse. Mais surtout, c'est la famille comme être collectif que cette dispersion périodique réduit à rien. Autrefois, la société domestique n'était pas seulement un assemblage d'individus, unis entre eux par des liens d'affection mutuelle; c'était aussi le groupe lui-même, dans son unité abstraite, impersonnelle. C'était le nom héréditaire avec tous les souvenirs qu'il rappelait, la maison familiale, le champ des aïeux, la situation et la réputation traditionnelles, etc. Tout cela tend à disparaitre. Une société qui se dissout à chaque instant pour se reformer avec d'autres éléments n'a pas assez de continuité pour se faire une physionomie personnelle, une histoire qui lui soit propre et à laquelle puissent s'attacher ses membres. Si donc les hommes ne remplacent pas cet ancien objectif de leur activité à mesure qu'il se dérobe à eux, il est impossible qu'il ne produise pas un grand vide dans l'existence.

Quand chaque milieu local était plus ou moins fermé aux autres par les usages, par les traditions, par la rareté des voies de communication, chaque génération était forcément retenue dans son milieu d'origine ou, tout au moins, ne pouvait pas s'en éloigner beaucoup. Mais à mesure que ces barrières s'abaissent, que ces milieux particuliers se nivellent et se perdent les uns dans les autres, il est inévitable que les individus se répandent, au gré de leurs ambitions et au mieux de leurs intérêts, dans les espaces plus vastes qui leur sont ouverts. Quant à la société religieuse, elle aussi n'est pas moins impropre à cette fonction. Ce n'est pas, sans 
doute, qu'elle n'ait pu, dans des conditions données, exercer une bienfaisante influence, mais c'est que les conditions nécessaires à cette influence ne sont plus actuellement données. On s'attendrait à ce que la société religieuse ait encore une bonne influence comme autrefois.

Si la religion, la famille, la patrie préservent du suicide égoïste, la cause n'en doit pas être recherchée dans la nature spéciale des sentiments que chacune met en jeu. Mais elles doivent toute cette vertu au fait général qu'elles sont des sociétés et elles ne l'ont que dans la mesure où elles sont des sociétés bien intégrées, c'est-à-dire sans excès ni dans un sens ni dans l'autre. Un tout autre groupe peut donc avoir la même action, pourvu qu'il ait la même cohésion.

En dehors de la société confessionnelle, familiale, politique, il est une autre sur laquelle nous voudrions attirer l'attention : c'est celle que forment, par leur association, y compris toute association jeunesse, tous les travailleurs du même ordre, tous les coopérateurs de la même fonction, c'est le groupe professionnel ou la corporation. Elle est apte à jouer ce rôle puisque, par définition, elle est composée d'individus qui se livrent aux mêmes travaux et dont les intérêts sont solidaires. Il n'est pas de terrain plus propice à la formation d'idées et de sentiments sociaux. L'identité d'origine, de culture, d'occupations fait de l'activité professionnelle la plus riche matière pour une vie commune. Par ailleurs, le groupe professionnel a sur tous les autres ce triple avantage qu'il est de tous les instants, de tous les lieux et que le pouvoir qu'il exerce s'étend à la plus grande partie de l'existence. La corporation a donc tout ce qu'il faut pour encadrer l'individu, pour le tirer de son état d'isolement moral et, étant donné l'insuffisance actuelle des autres groupes, elle est seule à pouvoir remplir cet indispensable office.

\section{Bibliographie}

ASSOCIATION QUÉBÉCOISE DE SUICIDOLOGIE (1999), «La semaine provinciale de prévention du suicide», Le Dossier Hommes et Suicide, http://www.cam.org/aqs/docs/ cahier hommes.html 
BIBEAU, G. et al. (1992), La santé mentale et ses visages. Un Québec pluriethnique au quotidien, Boucherville (Québec), Gaétan Morin.

BOUCHARD, G. (s.d.), Le suicide à l'adolescence, PsychoMédia.qc.ca http://www.psychomedia.qc.ca/dart7.htm

CACCIA, F. (1994), «Le suicide, un problème social», Le Devoir, 5 mai 1994, Montréal (Québec).

CHABOT, M. (1999), «Pourquoi tant d'hommes se suicident-ils?» Le Dossier Hommes et Suicide, http://www.cam.org/aqs/docs/cahier_hommes.html

CHAGNON, F. (1999), "Facteurs de risque du suicide chez les hommes selon l'âge», Le Dossier Hommes et Suicide, http://www.cam.org/aqs/docs/cahier hommes.html

CORIN, E. et al. (1985), La santé mentale du Québec. De la biologie à la culture, Comité de la santé mentale du Québec, Gouvernement du Québec.

DULAC, G. (1999), «La socialisation : un facteur inhibiteur de la recherche et de la demande d'aide chez les hommes», Le Dossier Hommes et Suicide, http://www.cam.org/aqs/docs/ cahier hommeshtml

DURKHEIM, E. (1969), Le suicide. Étude de sociologie, Paris, Presses Universitaires de France.

DYCK, R.J.et al. (1999), Forum national sur la santé. La santé et ses déterminants sommaires. Le suicide chez les enfants, les adolescents et les personnes âgées : constatations clés et mesures préconisées, http://wwwnfh.hc-sc.gc.ca/publicat/execsumm/dyckf.htm

LAMARTINE (de), A. (1960), Raphaël, Paris, Éditions Garnier Frères, 137-138.

LEFÈBVRE, S. (1998), «Le suicide des jeunes adultes : solitude et impuissance», Le Devoir, 7 février 1998, Montréal (Québec).

LEVINE, A. (1980), When Dreams and Heroes Die. A portrait of today's College students: Prepared for the Carnegie Council on Policy studies in Higher Education, $1^{\text {st }}$ ed., San Francisco, CA; Jossey-Bass, (New York), Carnegie Foundation for the Advancement of Teaching.

TOUSIGNANT, M. (1995), «Le suicide et les comportements suicidaires», dans Fernand Dumont, Simon Langlois et Yves Martin (sous la dir. de), Traité des problèmes sociaux, Institut québécois de recherche sur la culture. 